\title{
Historein
}

Vol 3 (2001)

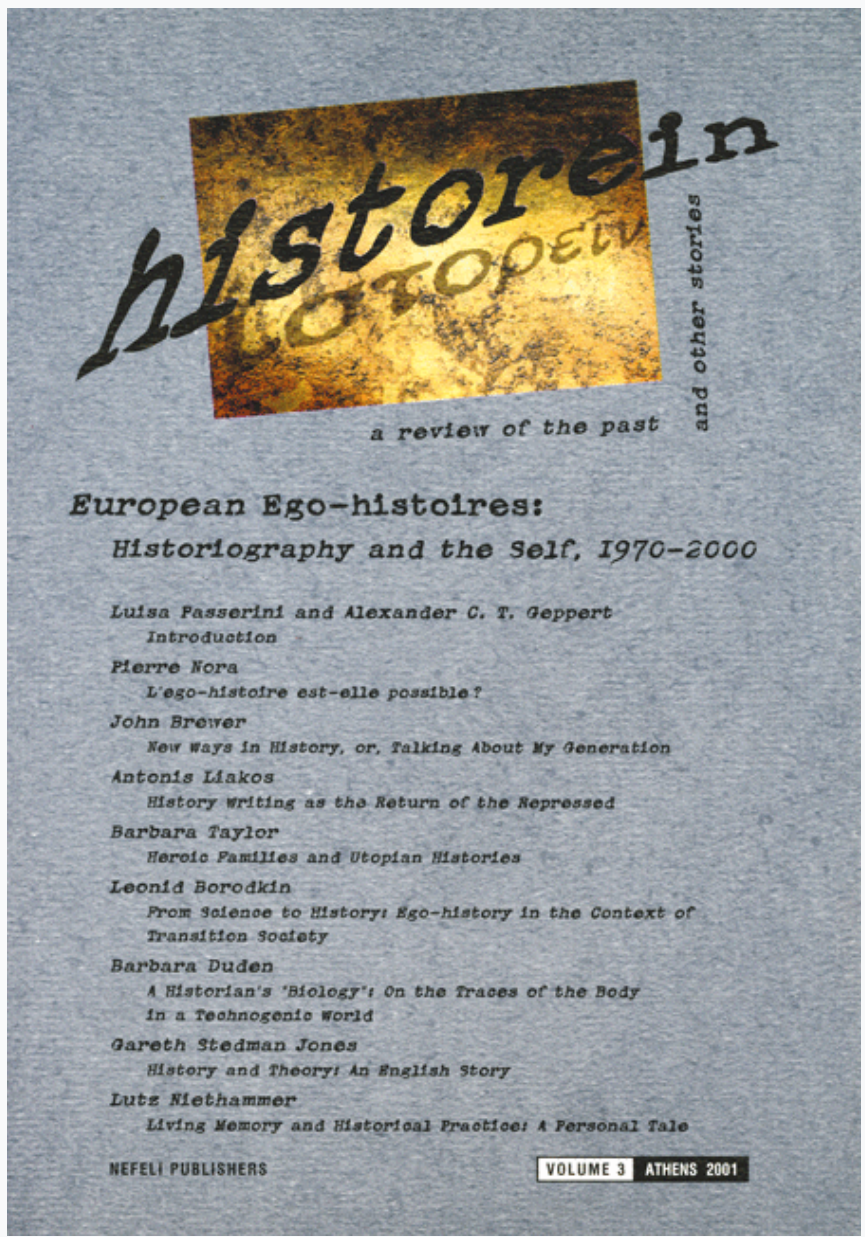

\section{Historians and (Auto)Biography: A Select Bibliography}

Alexander C. T. Geppert

doi: $\underline{10.12681 / \text { historein.105 }}$

\section{(2) 1090}

This work is licensed under a Creative Commons Attribution-NonCommercialShareAlike 4.0.

\section{To cite this article:}

Geppert, A. C. T. (2002). Historians and (Auto)Biography: A Select Bibliography. Historein, 3, 173-178. https://doi.org/10.12681/historein.105 


\section{Historians and (Auto)Biography: A Select Bibliography by Alexander C. T. Geppert}

\section{History, Autobiography and Ego-histoire}

Autour de l'ego-histoire, Le Débat, 49 (1988), pp. 122-40.

Barkin, Kenneth D., “Autobiography and History," Societas, 6 (1976), pp. 83-108.

Berg, Nicolas, "Zwischen individuellem und historiographischem Gedächtnis. Der Nationalsozialismus in Autobiographien deutscher Historiker nach 1945," BIOS, 13 (2000), pp. 181-207.

Bloch, Marc, Apologie pour l'histoire. Ou métier d'historien. Paris: A. Colin, 1993.

Bourdieu, Pierre, "L'lllusion biographique," Actes de la recherches en sciences sociales, 62/63 (1986), pp. 69-72.

Febvre, Lucien, Combats pour l'histoire. Paris: A. Colin, 1953.

Galtung, Johan, "Struktur, Kultur und intellektueller Stil. Ein vergleichender Essay über sachsonische, teutonische, gallische und nipponische Wissenschaft," Leviathan, 11 (1983), pp. 303-38.

Geppert, Alexander C. T., "Forschungstechnik oder historische Disziplin? Methodische Probleme der Oral History," Geschichte in Wissenschaft und Unterricht, 45 (1994), pp. 303-23.

Grass, Nikolaus, Wissenschaftsgeschichte in Lebensläufen. Hildesheim: Weidmann, 2001.

Klausa, Ekkehard, "Vom Gruppenbewußtsein akademischer Subkulturen. Deutsche Fakultäten um 1900. Ein inhaltsanalytischer Vorstoß in wissenschaftssoziologischer Absicht," Kölner Zeitschrift für Soziologie und Sozialpsychologie, 33 (1981), pp. 329-44.

Kohli, Martin, "'Von uns selber schweigen wir.' Wissenschaftsgeschichte aus Lebensgeschichten," in Wolf Lepenies (ed.), Geschichte der Soziologie. Studien zur kognitiven, sozialen und historischen Identität einer Disziplin. Vol.1. Frankfurt am Main: Suhrkamp, 1981, pp. 428-65.

Loewenberg, Peter, "Historical Method, the Subjectivity of the Researcher, and Psychohistory," The Psychohistory Review, 14 (1985), pp. 30-5.

Martin, Luther H., Gutman, Huck and Hutton, Patrick H. (eds.), Technologies of the Self. A Seminar with Michel Foucault. London: Tavistock, 1988.

Misch, Georg, Geschichte der Autobiographie. 4 vols. Frankfurt am Main: Schulte-Bulmke, 1962-76.

Molho, Anthony and Wood, Gordon S. (eds.), Imagined Histories. American Historians Interpret the Past. Princeton NJ: Princeton University Press, 1998.

Müller, Albert, "Alte Herren/Alte Meister. 'Ego-Histoire' in der österreichischen Geschichtswissenschaft. Eine Quellenkunde,” Österreichische Zeitschrift für Geschichtswissenschaften, 4 (1993), pp. 120-33.

Murphy, John, "The Voice of Memory. History, Autobiography and Oral Memory," Historical Studies, 22 (1986), pp. 157-75.

Niethammer, Lutz (ed.), Lebenserfahrung und kollektives Gedächtnis. Die Praxis der "Oral History." Frankfurt am Main: Suhrkamp, 1985.

Niethammer, Lutz, "Kommentar zu Pierre Bourdieu: Die biographische Illusion," BIOS, 3 (1990), pp. 91-3. Niggl, Günter, Geschichte der deutschen Autobiographie im 18. Jahrhundert. Theoretische Grundlegung und literarische Entfaltung. Stuttgart: Metzler, 1977. 
Niggl, Günter (ed.), Die Autobiographie. Zur Form und Geschichte einer literarischen Gattung. 2nd ed. Darmstadt: Wissenschaftliche Buchgesellschaft, 1998.

Novick, Peter, That Noble Dream. The "Objectivity Question" and the American Historical Profession. Cambridge: Cambridge University Press, 1988.

Passerini, Luisa, "Scrittura come trascendimento," Ventesimo Secolo, 1 (1991), pp. 63-7.

Popkin, Jeremy D., "Ego-histoire and Beyond. Contemporary French Historian-Autobiographers," French Historical Studies, 19 (1996), pp. 1139-67.

Popkin, Jeremy D., "Historians on the Autobiographical Frontier," American Historical Review, 104 (1999), pp. 725-48.

Redlich, Fritz, "Autobiographies as Sources for Social History. A Research Program," Vierteljahrschrift für Sozial- und Wirtschaftsgeschichte, 62 (1975), pp. 380-90.

Röckelein, Hedwig (ed.), Biographie als Geschichte. Tübingen: edition diskord, 1983.

Rosenthal, Gabriele, Erlebte und erzählte Lebensgeschichte. Gestalt und Struktur biographischer Lebensbeschreibungen. Frankfurt am Main/New York: Campus, 1995.

Wehler, Hans-Ulrich (ed.), Deutsche Historiker. 9 vols. Göttingen: Vandenhoeck \& Ruprecht, 1971-82.

\section{Autobiographical Productions by Historians}

Alter, Peter (ed.), Out of the Third Reich. Refugee Historians in Postwar Britain. London/New York: I.B. Tauris, 1998 [with contributions by Julius Carlebach, Francis L. Carsten, Edgar J. Feuchtwanger, J.A.S. Grenville, E.P. Hennock, Helmut Koenigsberger, Wolf Mendl, Werner E. Mosse, Helmut Pappe, Arnold Paucker, Sidney Pollard, Peter Pulzer, Nicholai Rubinstein, Walter Ullmann].

Ariès, Philippe, Un Historien du dimanche. Paris: Editions du Seuil, 1980.

Baltl, Hermann, Grass, Nikolaus and Faussner, Hans Constantin (eds.), Recht und Geschichte. Ein Beitrag zur österreichischen Gesellschafts- und Geistesgeschichte unserer Zeit. Zwanzig Historiker und Juristen berichten aus ihrem Leben. Sigmaringen: Jan Thorbecke, 1990 [with contributions by Heinrich Appelt, Hermann Baltl, Heinrich Fichtenau, Robert Göbl, Walter Goldinger, Nikolaus Grass, Friedrich Hausmann, Max Kaser, Walter Kastner, Hans Klecatsky, Erwin Melichar, Fritz Posch, Heinrich Schmidinger, Fritz Schwind, Ignatz Seidl-Hohenveldern, Walter Ullmann, Hermann Vetters, Hermann Wiesflecker, Richard Wolfram, Erich Zöllner].

Bloch, Marc, L'Étrange défaite. Témoignage écrit en 1940, suivi de ecrits clandestins 1942-1944. Paris: Michel, 1957.

Besançon, Alain, Une Génération. Paris: Julliard, 1987.

Braudel, Fernand, "Personal Testimony," Journal of Modern History, 44 (1972), pp. 448-67.

Croce, Benedetto, Contributo alla critica di me stesso. 2nd ed. Milano: Adelphi, 1989.

Cobb, Richard, The End of the Line. A Memoir. London: Murray, 1997.

Debouzy, Marianne, "From American Studies to American History. A French Point of View," The Journal of American History, 79 (1992), pp. 491-8.

Duby, Georges, L'Histoire continue. Paris: Jacob, 1991.

Fairbank, John K., Chinabound. A Fifty-Year Memoir. New York: Harper \& Row, 1982.

Fraser, Ronald, In Search of a Past. The Manor House, Amnersfield 1933-1945. London: Verso, 1984.

Friedländer, Saul, Quand vient le souvenir. Paris: Editions du Seuil, 1981. 
Galli, Giorgio, Passato prossimo. Persone e incontri 1949-1999. Milano: Kaos, 2000.

Galli della Loggia, Ernesto, Vent'anni d'impazienza. Interventi 1976-1998. Scritti di politica e di battaglia. Firenze: Liberal Libri, 2001.

Gay, Peter; My German Question. Growing Up in Nazi Berlin. New Haven: Yale University Press, 1998. Gibbon, Edward, Memoirs of My Life. Harmondsworth: Penguin, 1984.

Girardet, Raoul, Singulièrement libre. Entretiens. Paris: Perrin, 1990.

Grass, Nikolaus (ed.), Österreichische Geschichtswissenschaft der Gegenwart in Selbstdarstellungen. 2 vols. Innsbruck: Wagner, 1950-51 [with contributions by Heinrich Hammer, Richard Heuberger, August Loehr, Franz Martin, Hans Pirchegger, Otto Stolz, Josef Weingartner, Leopold Wenger, Hermann Wopfner, Anton Dörrer, Dagobert Frey, Viktor Geramb, Rudolf Köstler, Paul Koschaker, Hans Planitz, Max Rintelen, Leo Santifaller, Heinrich Felix Schmid, Mathilde Uhlirz, Ignaz Zibermayr].

Greenberg, Douglas and Katz, Stanley N. (eds.), The Life of Learning. The Charles Homer Haskins Lectures of the American Council of Learned Societies. New York: Oxford University Press, 1994 [with contributions by Maynard Mack, Mary Rosamond Haas, Lawrence Stone, Milton V. Anastos, Carl E. Schorske, John Hope Franklin, Judith N. Sklar, Paul Oskar Kristeller, Milton Babbit, Donald W. Meinig, Annemarie Schimmel].

Haffner, Sebastian, Geschichte eines Deutschen. Die Erinnerungen 1914-1933. Stuttgart: Deutsche Verlags-Anstalt, 2000.

Huizinga, Johan, "Mein Weg zur Geschichte," in Johan Huizinga, Mein Weg zur Geschichte. Letzte Reden und Skizzen. Basel: Schwabe, 1947, pp. 9-60.

Isnenghi, Mario, "Minima Personalia," Belfagor, (1988).

Jacobeit, Wolfgang, Von West nach Ost - und zurück. Autobiographisches eines Grenzgängers zwischen Tradition und Innovation. Münster: Westfälisches Dampfboot, 2000.

Katz, Jacob, With My Own Eyes. The Autobiography of an Historian. Hanover, NH: University Press of New England for Brandeis University Press, 1995.

Klein, Fritz, Drinnen und Draußen. Ein Historiker in der DDR. Erinnerungen. Frankfurt am Main: S. Fischer, 2000.

Kriegel, Annie, Ce que j'ai cru comprendre. Paris: Laffont, 1991.

Kuczynski, Jürgen, Ein Leben in der Wissenschaft der DDR. Münster: Westtälisches Dampfboot, 1994.

Le Goff, Jacques, Une vie pour l'histoire. Entretiens avec Marc Heurgon. Paris: La Découverte, 1996.

Le Roy Ladurie, Emmanuel, Paris-Montpellier. P.C.-P.S.U., 1945-1963. Paris: Gallimard, 1982.

Lehmann, Hartmut and Oexle, Otto Gerhard (eds.), Erinnerungsstücke. Wege in die Vergangenheit. Rudolf Vierhaus zum 75. Geburtstag gewidmet. Köln/Weimar: Böhlau, 1997 [with contributions by Karl Otmar Freiherr von Aretin, Karl Dietrich Bracher, Fritz Fellner, Iring Fetscher, Klaus Friedland, Irmgard Höß, Walther Hofer, Erich Kosthorst, Annelise Thimme, Eberhard Weis, Karl Ferdinand Werner und Wolfgang Zorn].

Lehmann, Hartmut and Sheehan, James J. (eds.), An Interrupted Past. German-Speaking Refugee Historians in the United States after 1933. New York: Cambridge University Press/German Historical Institute Washington DC, 1991.

Medick, Hans, "Rede zur Verleihung des René-Kuczynski-Preises 1997," 1999, 14 (1999), pp. 191-200. Meinecke, Friedrich, Erlebtes 1862-1901. Leipzig: Koehler \& Amelang, 1941. 
Meyer, Jean (ed.), Egohistorias: El amor a Clio. México: Centre d'études mexicaines et centreaméricaines, 1993 [with contributions by Antonio Alatorre, Miguel Léon-Portilla, Octavio Paz, Luis Villoro, Silivio Zavala, Alfredo López Austin and Edmundo 0’Gorman].

Mosse, George, “ch bleibe Emigrant.” Gespräche mit George L. Mosse. Berlin: Dietz, 1991.

Mosse, George L., Confronting History. A Memoir. Madison, Wl: University of Wisconsin Press, 2000. Mumford, Lewis, Sketches from Life. The Autobiography of Lewis Mumford. The Early Years. New York: Dial Press, 1982

Nipperdey, Thomas, "Eine bürgerliche Jugend (1927-1945)," in Der Äquadukt 1763-1988. Ein Almanach aus dem Verlag C.H. Beck im 225. Jahr seines Bestehens. München: C.H. Beck, 1988, pp. 126-43.

Nora, Pierre (ed.), Essais d'ego-histoire. Paris: Gallimard, 1987 [with contributions by Maurice Agulhon, Pierre Chaunu, Georges Duby, Raoul Girardet, Jacques Le Goff, Michelle Perrot, René Rémond].

Passerini, Luisa, Autoritratto di gruppo. Firenze: Giunti, 1988 [Autobiography of a Generation. Italy, 1968. Hanover/London: Wesleyan University Press, 1996].

Petzold, Joachim, Parteinahme wofür? Historiker im Spannungsfeld von Politik und Wissenschaft. Berlin: Verlag für Berlin-Brandenburg, 2001.

Pohl, Karl Heinrich (ed.), Historiker in der DDR. Göttingen: Vandenhoeck \& Ruprecht, 1997.

Richert, Dominik, Beste Gelegenheit zum Sterben. Meine Erlebnisse im Krieg 1914-1918. München: Knesebeck \& Schuler, 1989.

Schorske, Carl. E., A Life of Learning. Charles Homer Haskins Lecture for 1987. New York, NY: American Council of Learned Societies, 1987 (http://www.acls.org./op1.htm).

Schulz-Hageleit, Peter, Leben in Deutschland, 1900-1950. Historisch-psychoanalytische Betrachtungen. Pfaffenweiler: Centaurus, 1994.

Schulz-Hageleit, Peter, Leben in Deutschland, 1945-1995. Geschichtsanalytische Reflexionen. Pfaffenweiler: Centaurus, 1996.

Schulz-Hageleit, Peter, Leben in Deutschland. Geschichtsanalytische Reflexionen über Gegenwart und Zukunft. Pfaffenweiler: Centaurus, 1998.

Steedman, Carolyn Kay, Landscape for a Good Woman. A Story of Two Lives. New Brunswick, NJ: Rutgers University Press, 1986.

Steinberg, Sigfrid (ed.), Die Geschichtswissenschaft der Gegenwart in Selbstdarstellungen. 2 vols. Leipzig: Felix Meiner, 1925/26 [with contributions by Georg von Below, Alfons Dopsch, Heinrich Finke, Walter Goetz, R. F. Kaindl, Max Lehmann, Georg Steinhausen, Karl Julius Beloch, Harry Bresslau, Victor Gardthausen, George Peabody Gooch, Nicolaas Japikse, Ludwig Freiherr von Pastor, Felix Rachfahl].

Veyne, Paul, Le Quotidien et l'intéressant. Entretiens avec Catherine Darbo-Peschanski. Paris: Belles Lettres, 1995.

Vidal-Naquet, Pierre, Mémoires. Paris: Editions du Seuil, 1995.

Vivarelli, Roberto, La fine di una stagione. Memoria 1943-1945. Bologna: il Mulino, 2000.

Zemon Davis, Natalie, A Life of Learning. Charles Homer Haskins Lecture for 1997. New York, NY: American Council of Learned Societies, 1997 (http://www.acls.org./op39.htm). 


\section{Interviews with Historians*}

Abelson, Elaine, "Interview with Joan Scott," Radical History Review, 45 (1989), pp. 41-59.

Adelson, Roger (ed.), Speaking of History. Conversations with Historians. East Lansing: Michigan

State University Press, 1997 [interviews with Walter L. Arnstein, Thomas D. Clark, Natalie Zemon

Davis, John Demos, Gilbert C. Fite, Darlene Clark Hine, Joan M. Jensen, Afaf Lutfi Al-Sayyid Marsot, William H. McNeill, Helen Nader, Stephen J. Pyne, Theodore H. Von Laue, C. Vann Woodward].

Adelson, Roger, “Interview with Caroline Walker Bynum,” The Historian, 59 (1996), pp. 1-17.

Adelson, Roger, "Interview with Frederick C. Wakeman," The Historian, 59 (1997), pp. 505-21.

Adelson, Roger, "Interview with Mary Beth Norton," The Historian, 60 (1997), pp. 1-19.

Adelson, Roger, "Interview with Robin Higham," The Historian, 60 (1998), pp. 473-486.

Adelson, Roger, "Interview with Asuncion Lavrin," The Historian, 61 (1998), pp. 1-14.

Adelson, Roger, “Interview with Michael J. Hogan," The Historian, 61(1999), pp. 502-17.

Adelson, Roger, "Interview with Carol Gluck," The Historian, 62 (1999), pp. 1-16.

Adelson, Roger, “Interview with Wm. Roger Louis," The Historian, 62 (2000), pp. 493-509.

Adelson, Roger, “Interview with Sara Margaret Evans," The Historian, 63 (2000), pp. 1-15.

Adelson, Roger, "Interview with Peter Iverson," The Historian, 63 (2001), pp. 491-504.

Adelson, Roger, "Interview with James H. Billington," The Historian, 64 (2001), pp. 1-17.

Adelson, Roger and Smith, Russell, "Videotaped Interviews with British Historians, 1985-1998," Albion, 31 (1999), pp. 257-68.

Archambault, Paul, “An Interview with Jacques Le Goff," Historical Reflections, 21 (1995), pp. 155-85.

Aufderheide, Pat, "Interview with Natalie Davis," Radical History Review, 28-30 (1984), pp. 136-9.

Bejczy, Istvan, "Wir schauen in einen Spiegel und sehen einen anderen. Gespräch mit Frank Ankersmit," Österreichische Zeitschrift für Geschichtswissenschaften, 4 (1993), pp. 457-65.

Coppin, Judy and Harding, Robert, "Intervista a Natalie Zemon Davis," Memoria: Rivista di Storia delle Donne, 9 (1983), pp. 79-93.

Delanoë, Nelcya, "France Celebrates the Bicentennial. Interview with Madeleine Réberioux," Radical History Review, 48 (1990), pp. 134-41.

Dipper, Christof, "Begriffsgeschichte, Sozialgeschichte, begriffene Geschichte. Reinhart Koselleck im Gespräch mit Christof Dipper," Neue Politische Literatur, 43 (1998), pp. 187-205.

Döcker, Ulrike and Sieder, Reinhard, "Deutschlands Historiker/innen nach dem Fall der Mauer. Ein Gespräch mit Jürgen Kocka (Berlin)," Österreichische Zeitschrift für Geschichtswissenschaften, 3 (1992), pp. 65-75.

Domanska, Ewa, "Human Face of Scientific Mind. An Interview with Hayden White," Storia della Storiografia, 24 (1993), pp. 5-21.

Evans, Richard J., Hobsbawm, Eric and Müller, Albert, "Die Verteidigung der Geschichte," Österreichische Zeitschrift für Geschichtswissenschaften, 9 (1998), pp. 107-23.

Frader, Laura and De Grazia, Victoria, "New Subjects, New Social Commitments. An Interview with Michelle Perrot," Radical History Review, 37 (1987), pp. 27-38.

Hohls, Rüdiger, Jarausch, Konrad H. and Hacke, Jens (eds.), Versäumte Fragen. Deutsche Historiker im Schatten des Nationalsozialismus. Stuttgart: Deutsche Verlags-Anstalt, 2000 [interviews with 
Rudolf Vierhaus, Wolfram Fischer, Gerhard A. Ritter, Helga Grebing, Hans Mommsen, Wolfgang J. Mommsen, Imanuel Geiss, Hans-Ulrich Wehler, Reinhard Rürup, Wolfgang Schieder, Lothar Gall, Hartmut Lehmann, Adelheid von Saldern, Michael Stürmer, Heinrich August Winkler. Jürgen Kocka, Winfried Schulze].

"Im Gespräch: Edith Saurer and Hans Medick," L'Homme, 7 (1996), pp. 70-86.

Koufou, Angelica and Miliori, Margarita, "The Ironic Poetics of Late Modernity. An Interview with Hayden White," Historein, 2 (2000), pp. 183-200.

"Kultureller Nationalismus in einem Europa der Regionen. Ein Gespräch mit Ernest Gellner," Österreichische Zeitschrift für Geschichtswissenschaften, 4 (1993), pp. 137-45.

Luria, Keith and Gandolfo, Romulo, "Carlo Ginzburg. An Interview," Radical History Review, 35 (1986), pp. 89-111.

MARHO: The Radical Historians Organization (ed.), Visions of History. New York: Pantheon Books, 1984 [interviews with E. P. Thompson, Eric Hobsbawm, Sheila Rowbotham, Linda Gordon, Natalie Zemon Davis, William Appleman Williams, Staughton Lund, Danid Montgomery, Herbert Gutman, Vincent Harding, John Womack, C. L. R. James, Moshe Lewin].

Murrell, Gary, "On Herbert Aptheker and His Side of History. An Interview with Eric Foner," in Radical History Review, 78 (2000), pp. 5-26.

Noiriel, Gérard, “L'Histoire culturelle aujourd'hui. Entretien avec Roger Chartier," Genèses, 15 (1994), pp. 115-29.

Rüsen, Jörn, "Modernität der Historie. Zu neuen Ansätzen einer Geschichte der Historiographie," Österreichische Zeitschrift für Geschichtswissenschaften, 2 (1991), pp. 90-5.

Sassen, Saskia, "We are not done with the State," Österreichische Zeitschrift für Geschichtswissenschaften, 11 (2000), pp. 129-38.

Stave, Bruce M., "A Conversation with Anthony R. Sutcliffe," Journal of Urban History, 7 (1981), pp. 335-79.

Thane, Pat and Lunbeck, Liz, "An Interview with Eric Hobsbawm," Radical History Review, 19 (1979), pp. 111-31.

Thane, Pat, "Interviews with Historians," The Historian, 36 (1992), pp. 18-20.

Walker, Pamela J., "Interview with Dorothy Thompson," Radical History Review, 77 (2000), pp. 4-19. Wallace, Mike, "Interview with Nicaraguan Historians," Radical History Review, 33 (1985), pp. 7-20.

White, Hayden, "Ich glaube nicht, daß eine Theorie wie meine dazu da ist, angewandt zu werden," Österreichische Zeitschrift für Geschichtswissenschaften, 9 (1998), pp. 246-55.

Wolf, Eric R., "Anthropologie und Geschichte," Österreichische Zeitschrift für Geschichtswissenschaften, 9 (1998), pp. 256-68.

*Journals such as Radical History Review, Hispanic American Historical Review, The Historian and Österreichische Zeitschrift für Geschichtswissenschaften regularly feature interviews with historians of which only a few can be listed here. 